\title{
Commentary: Developmental delay and congenital heart disease: Insights from a novel "artificial womb" model
}

Nobuyuki Ishibashi, MD, and Richard A. Jonas, MD

\author{
From the Division of Cardiac Surgery, Children's National Medical Center, Washington, DC. \\ Disclosures: Authors have nothing to disclose with regard to commercial support. \\ Received for publication Jan 8, 2019; accepted for publication Jan 8, 2019; available ahead of print Feb 16, 2019. \\ Address for reprints: Richard A. Jonas, MD, Department of Cardiovascular Surgery, Children's National Medical \\ Center, 111 Michigan Ave NW, Washington, DC 20010 (E-mail: rjonas@childrensnational.org). \\ J Thorac Cardiovasc Surg 2019;157:1992-3 \\ $0022-5223 / \$ 36.00$ \\ Copyright (c) 2019 by The American Association for Thoracic Surgery \\ https://doi.org/10.1016/j.jtcvs.2019.01.031
}

The article by Lawrence and colleagues ${ }^{1}$ from Children's Hospital of Philadelphia in this issue of the Journal describes an interesting and innovative laboratory study. The authors have applied the "artificial womb" that has been developed by the fetal research group at the Children's Hospital of Philadelphia. This is a closed system that circulates an artificial amniotic fluid and supplies parenteral nutrition to a fetal lamb. It achieves gas transfer using a simple neonatal oxygenator connected to the umbilical vessels with no pump support other than the fetal circulation. The animals remain hemodynamically stable presumably facilitated by the low resistance of the oxygenator. This socalled EXTEND system has recently been documented to allow ex utero fetal growth of severely premature animals over approximately a 30-day period, suggesting that it could be clinically applicable for babies who are born severely prematurely.

In this study using the EXTEND system, Lawrence and colleagues ${ }^{1}$ have compared brain development under conditions of normoxic oxygen delivery versus hypoxia. Their goal is to simulate the hypoxic cerebral circulation that occurs prenatally in several (although not all) congenital heart anomalies. The authors have concluded that chronic fetal hypoxia results in a number of abnormalities of brain development, including white matter hypovascularity and impaired myelination. These findings are consistent with clinical observations using fetal magnetic resonance imaging suggesting reduced brain growth, particularly white matter in the fetus with congenital heart disease. The study adds further support to the hypothesis that intracerebral hypoxia as a consequence of altered circulation is one underlying cause of delayed brain development in the fetus with congenital heart disease. However, the model does not allow exploring additional mechanisms of brain underdevelopment and delayed maturation, including changes in cerebral blood flow induced by a congenital heart anomaly or, even more important, the underlying genetic basis of the heart problem. For example, it is well known that cells from the neural crest play a key role in conotruncal, semilunar valve, and brain development. Di George syndrome is an

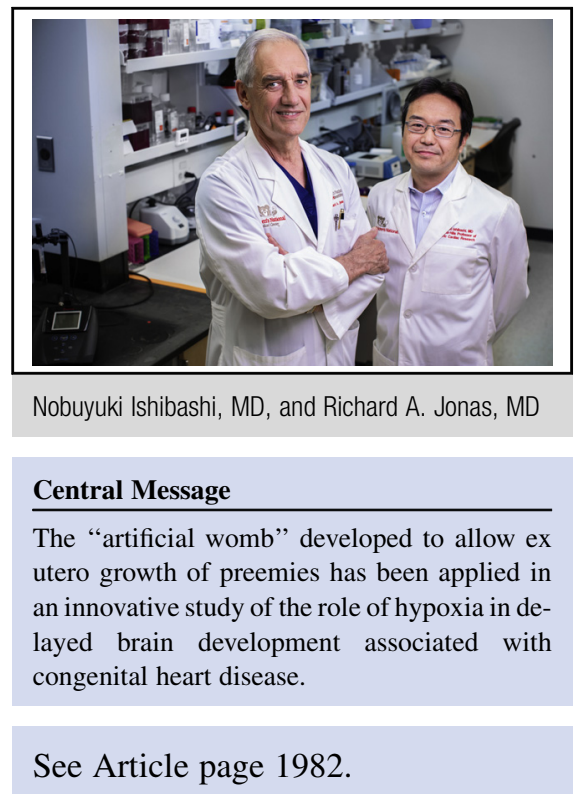

example of a neural crest constellation of anomalies caused by deletion of 30 to 50 genes from the long arm of chromosome 22. It is associated with not only multiple cardiac anomalies but also moderately severe developmental delay and psychiatric illness. As recent advances in genome editing, such as CRISPR/Cas9, become more available in larger mammals, it should be feasible to generate preclinical large animal models to access mechanistic questions related to the cumulative and complex effects of congenital heart disease on key developmental events.

One of the limitations of this study ${ }^{1}$ is that although there were differences in brain weight between the normoxic and hypoxic fetuses and control animals that were not supported with the EXTEND system, there was no difference between the normoxic and hypoxic groups themselves in cortical, cerebellar, and total brain weight. This is important because a global reduction in brain size and weight is a key feature of the fetal and neonatal brain with congenital heart disease. Table 2 suggests that it is the EXTEND system that leads to lower brain weight rather than hypoxia. Therefore, improvement of the system will offer a unique opportunity for elucidating the impact of fetal hypoxia on the developing brain.

Lawrence and colleagues ${ }^{1}$ found reduced numbers of neurons in the white matter and CA1 in the hypoxic group and speculated that impaired neuronal migration might be 
involved. However, their method using Luxol fast blue with cresyl violet staining is not suited to defining migrating cell populations. Additional immunohistochemistry studies to define the migrating cell population are needed. Other possible explanations of the reduced numbers of neurons include increased nonapoptotic cell death or reduced proliferation. Further application of other cutting-edge neuroscience methods will be helpful in determining the complex cellular mechanisms underlying the brain injury seen in children with congenital heart disease.
Lawrence and colleagues ${ }^{1}$ are to be congratulated in applying a novel fetal model in studying an area of great interest in the field of congenital heart disease, namely, the mechanisms of delayed brain development associated with many cardiac anomalies.

\section{Reference}

1. Lawrence KM, McGovern PE, Mejaddam A, Rossidis AC, Baumgarten H, Kim A, et al. Chronic intrauterine hypoxia alters neurodevelopment in fetal sheep. J Thorac Cardiovasc Surg. 2019;157:1982-91. 